Casos Clínicos

Arch. Esp. Urol., 61, 7 (831-834), 2008

\title{
TUMOR ADENOMATOIDE EPIDIDIMARIO APORTACIÓN DE 5 CASOS
}

Roberto Llarena Ibarguren, Jorge García-Olaverri Rodríguez, Ivan Olano Grasa, Igor Azurmendi Arín, Emilio Cantón Aller y Carlos Pertusa Peña.

Servicio de Urología. Hospital de Cruces. Bilbao. Vizcaya. España.

Resumen.- OBJETIVO: El tumor adenomatoide de epidídimo es infrecuente, de carácter benigno, no habiéndose descrito evoluciones malignas.

MÉTODO: Presentamos 5 casos, con edades variopintas entre los 31 y los 76 años, y también con tamaños diversos entre los 6 y los $30 \mathrm{~mm}$. Todos fueron intervenidos realizándose exéresis de la masa, tras varios meses de crecimiento. En un caso la evolución era de 6 años.

RESULTADO: Ninguno presentó ni recidiva ni evolución tórpida tras la cirugía. La histología confirmó el carácter adenomatoide benigno en todos ellos.

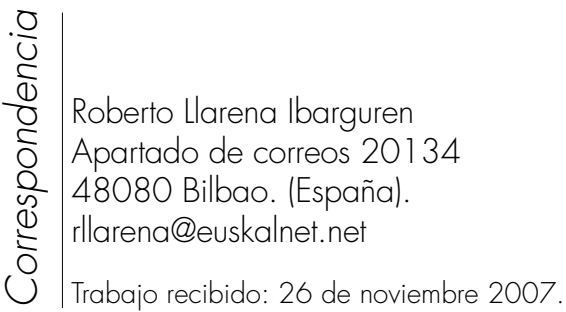


CONCLUSIONES: A pesar de que los hallazgos clínicos, ecográficos y exploratorios sugieren la localización epididimaria y la benignidad, es mandatoria la exploración y extirpación quirúrgica de toda masa sólida paratesticular.

\section{Palabras clave: Epidídimo. Tumor. Adenomatoide.}

Summary.- OBJECTIVE: Adenomatoid tumor of the epididymis is unfrequent, benign, with no malignant outcomes described.

METHODS: We report five cases, with patient's ages varying from 31 to 76 years, and tumor sizes from 6 to $30 \mathrm{~mm}$. All patients underwent surgery with excision of the mass, some of them after several months of growing. In one of the cases the evolution reached six years.

RESUITS: None of the cases presented recurrence or bad outcome after surgery. Pathology confirmed the benign adenomatoid character in all cases.

CONCLUSIONS: Despite the clinical, ultrasound and physical examination findings suggest the localization in the epididymis and its benign character, surgical exploration is mandatory with surgical excision of the paratesticular mass.

Keywords: Epididymis. Tumor. Adenomatoid.

\section{INTRODUCCIÓN}

Tumor benigno por definición, del que nunca se han descrito comportamientos malignos ni metástasis, el tumor adenomatoide de epidídimo (TA), es objeto de publicación esporádica, la mayoría de las veces con pocos casos.

Los más de 50 años que lleva el TA en la literatura lo hacen considerar como una tumoración benigna en su comportamiento y en su evolución, que anida en tejidos paratesticulares preferentemente epididimarios, $y$ en las trompas de Falopio femeninas (1).

Su denominación como adenomatoide hace referencia a su aspecto glandular (2).

En nuestro medio fue descrito por primera vez en 1968 por Montero en esta misma revista (2). Según Moyano (3) en abril de 2007 habían sido publicados unos 35 casos, lo que unidos al caso de Serrano (4) publicado en el mismo número que el de Moyano, y a los 2 últimos de Garrido (5) harán unos 38. Siempre que hablamos del total de casos presentados y reportados de una determinada patología hay que excusarse de antemano ante posibles aunque involuntarios olvidos.

\section{CASOS CLÍNICOS}

Presentamos 5 casos, 1 de ellos objeto de presentación en esta misma publicación en 1997 (1), acontecidos en varones de entre 31 y 76 años. Los tamaños variaron entre $6 \mathrm{~mm}$ y $30 \mathrm{~mm}$. Los 5 casos asentaron en cola de epidídimo. La evolución del proceso presentó asimismo amplia variación con un caso de hasta 6 años. La casuística se presenta en la Tabla I. En todos se procedió a exéresis del nódulo mediante cirugía transescrotal. Todos fueron informados histopatológicamente como tumor adenomatoide compuesto en mayor o menor cantidad por estructuras glandulares rodeadas de epitelio vacuolado, tejido conectivo estroma sin músculo liso y acúmulos linfoides.

La evolución tras la extirpación fue favorable en todos los pacientes, sin lamentar recidivas.

\section{DISCUSIÓN}

Se define el TA como aquel proceso neoformativo de carácter benigno de origen mesenquimal que asienta en el tracto urogenital. En el varón y como localización más frecuente es el epidídimo, sin olvidar citar el cordón

TABLA I. NUESTRA SERIE.

\begin{tabular}{|c|c|c|c|c|}
\hline & Edad & Evolución & Lado & Tamaño \\
\hline Caso 1 & 60 años & 2 años & Izdo polo inf & $6 \mathrm{~mm}$ \\
\hline Caso 2 & 31 años & 2 años & Izdo polo inf & $15 \mathrm{~mm}$ \\
\hline Caso 3 & 76 años & 6 años & Izdo polo inf & $25 \mathrm{~mm}$ \\
\hline Caso 4 & 63 años & 1 año & Dcho polo inf & $12 \mathrm{~mm}$ \\
\hline Caso 5 & 50 años & 18 meses & Dcho polo inf & $30 \mathrm{~mm}$ \\
\hline
\end{tabular}




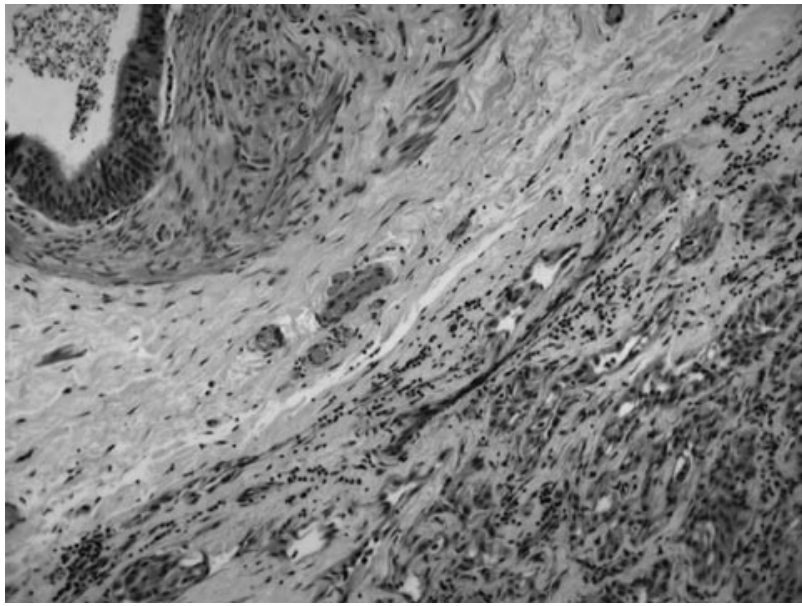

FIGURA 1. Estructuras glandulares rodeadas de epitelio vacuolado, tejido conectivo estromal sin músculo liso y acúmulos linfoides.

funicular, la albugínea testicular, deferentes, próstata y glándulas suprarrenales. En la mujer puede asentar en trompas, útero e hilio ovárico (3).

Según Pellicé (6) los tumores epididimarios pueden ser primarios de origen mesotelial o epitelial y secundarios o metástasicos. Los tumores localizados en epidídimo suponen menos del $5 \%$ de las neoplasias intraescrotales, representando los benignos el $75 \%$ de todos ellos, siendo el TA el más frecuente. El TA representa el 30\% de las masas paratesticulares, seguido del leiomioma y en menor medida el cistoadenoma papilar (6), mientras que los malignos más frecuentes, dentro de la rareza, serán sarcomas, metástasis sobre todo de estómago, páncreas incluso riñón, existiendo también alguna descripción de carcinoma primario (6).

Como ya hemos apuntado, el TA más frecuente es el de localización epididimaria, pudiendo asentar en cabeza - cola, aunque sea más frecuente su descubrimiento en ésta última (2-5). Acostumbra a presentarse como una tumoración firme e indolora, redondeada u ovalada, a veces de larga evolución, y con poca variación en su tamaño a lo largo del tiempo. Se han descrito entre los $5 \mathrm{~mm}$ y los $5 \mathrm{~cm}(5)$, aunque en general el tamaño ronda los $2 \mathrm{~cm}$.

Puede aparecer a cualquier edad, aunque es más común su descubrimiento entre los 30 y 50 años (1).

Se relaciona con la presencia de hidrocele hasta en un $50 \%$ de los casos. Asimismo en el $30 \%$ se asocian procesos intercurrentes inflamatorios o traumáticos, que ponen de manifiesto el TA, aunque no sean los causantes directos del TA. (7).

Se han descrito 3 formas de presentación: tumoral, en la que existe una clara diferenciación con el teste, in- flamatoria de difícil diagnóstico frente a la epdidimitis aguda o crónica, y la maligna en la que existe una mala y difícil diferenciación con el teste (6).

Macroscopicamente se trata de lesiones sólidas, de superficie fibrosa. Microscópicamente constan de una serie de espacios más o menos irregulares, revestidos por una capa de celulas epiteliales planas o cúbicas rodeadas de estroma colágeno e incluso fibras musculares y elásticas (1). Cuando las bandas musculares son abundantes el tumor pasa a denominarse leiomioma adenomatoide (8). Muy frecuentemente se encuentran vacuolas en el interior de las células pudiendo estas llegar a ocupar todo el citoplasma. La disposición de las células epiteliales permite reconocer 3 patrones: plexiforme, glandular y angiomatoide (8).

Inmunohistoquimicamente expresa siempre citoqueratinas AE 1/AE3 y EMA, no expresando marcadores epiteliales ni factores VIII y CD34. $(3,5)$.

El TA es de origen incierto aunque se presupone un origen mesotelial, sin que esto explique su localización genital (9). La teoría del origen mesotelial lo da la tinción para calretinina, proteína fijadora del calcio perteneciente a la familia de la proteína S100 localizada tanto en el citoplasma como en el núcleo celular (3).

La palpación escrotal pondrá de manifiesto la existencia de una formación sólida, firme, a menudo indolora, situada como hemos comentado la mayoría de las veces en epidídimo, ya sea en cabeza o más frecuentemente en cola, de un tamaño variable entre los $5 \mathrm{~mm}$ pudiendo llegar a los $5 \mathrm{~cm}$.

Ecográficamente la neoformación se comporta como sólida, de ecogenicidad aumentada y casi siempre homogénea, diferenciándose la mayoría de las ocasiones del parénquima testicular, salvo en las formas de presentación definidas como inflamatorias o malignas en las que la distinción con el teste puede ser mas dificultosa.

Muchos autores preconizan el empleo confirmatorio de la resonancia magnética nuclear para el correcto diagnóstico, apareciendo el TA como una formación sólida hiperdensa, aunque nosotros no lo consideramos imprescindible, disponiendo de un resolutivo examen sonográfico.

El diagnóstico diferencial ha de establecerse tanto como formaciones liquidas, quiste de epidídimo, quiste funicular, espermatocele o hernia inguinoescrotal, o sólidas como el tumor testicular, procesos inflamatorios o infecciosos y hematoma intratesticular.

Una vez obtenido el diagnóstico la escrototomía nos facilitará el acceso a la tumoración, y la posterior exéresis que acostumbra a ser sencilla, cuando la neoformacion asienta en forma extratesticular, debiendo recurrir a la inguinotomía cuando el asiento sea dudoso o intratesti- 
cular, ante la sospecha razonable de un tumor testicular (1).

No están descritas recidivas, incluso en los raros casos que presentan atipias o invasión local $(1,5)$.

\section{BIBLIOGRAFÍA y LECTURAS RECOMENDADAS (*lectura de interés y lectura fundamental)}

*1. VESGA, F.; ACHA, M.; LLARENA, R. y cols.: "Tumor adenomatoide paratesticular". Arch. Esp. Urol., 50: 292, 1997.

**2. MONTERO, J.: "Tumor adenomatoide de epidídimo". Arch. Esp. Urol., 21: 425, 1968.

3. MOYANO, J.L.; GIRADLES, J.; SANCHEZ, J. y cols.: "Tumor adenomatoide de epidídimo". Actas Urol. Esp., 31: 417, 2007.

4. SERRANO, P.; MEDRANO, P.; BORQUE, A. y cols.: "Consulta por masa escrotal: Lesiones epididimarias". Actas Urol. Esp., 31: 420, 2007.

*5. GARRIDO, P.; JIMÉNEZ, M.; HERRANZ, L.M. y cols.: "Tumor adenomatoide de epidídimo. Aportación de dos casos". Arch. Esp. Urol., 60: 700, 2007.

*6. PELLICÉ, C.; COSME, M.; PÉREZ, S.: "Tumores adenomatoides de epidídimo. Dos nuevas aportaciones". Arch. Esp. Urol., 50: 127, 1997.

7. MONTSERRAT, V.; VESSA, J.; MONTANE, M. y cols.: "Tumores adenomatoides de epidídimo". Arch. Esp. Urol., 46: 725, 1993.

8. ROMANELLI, R. SANNA, A.: "Adenomatoid leiomyoma and papillary cystadenoma of the epididymis". Pathologica, 77: 445, 1985.

9. ALAPONT, F.M.; GÁLVEZ, J.; LAMBER, P.M. y cols.: "Tumor adenomatoide de epidídimo". Actas Urol. Esp., 23: 613, 1999. 